\title{
New Network Models With New Graph Labellings For Information Cryptograph
}

\author{
Hui Sun ${ }^{1}$, Xiaohui Zhang ${ }^{1}$, Bing Yao ${ }^{1, \dagger}$, Mingjun Zhang $^{2}$, Ming Yao $^{3}$ \\ 1. College of Mathematics and Statistics, Northwest Normal University, Lanzhou 730070, China \\ ${ }^{\dagger}$ Corresponding author: yybb918@163.com. \\ 2. School of Information Engineering Lanzhou University of Finance and Economics, Lanzhou, 730101 China \\ 3. Department of Information Process and Control Engineering, Lanzhou Petrochemical College of Vocational Technology \\ Lanzhou, 730060 China
}

Keywords: cryptograph; models; edge magic graceful labelling; graphical password; algorithm.

\begin{abstract}
Based on the idea of of "graphical construction plus number theory" and magic type of various labellings for solving network transfer delay by using other type of graphical passwords, we define a new graph labelling, called generalized edge magic graceful labelling, and build some large scale of network models having the new labellings to verify effective algorithms.
\end{abstract}

\section{Introduction}

\subsection{Researching background}

It is noticeable, the contesting control of internet keyresources and cyber-space in the world will be more intense, the network security risk of industrial control system, intelligent technology application, cloud computing and mobile payment domain will be further increased. It is imperative to study new passwords. Wang et al. [15] design some new graphical passwords by their idea of "graphical construction plus number theory" to aim new secure and memorable passwords for users and very difficult to be broken [14]. Suo et al. [13] point out: Knowledge based techniques are the most widely used authentication techniques and include both text-based and picture-based passwords. From considering storage and communication, graphical passwords require much more storage space than text based passwords. However, the graphical constructions proposed by Wang et al. are graphs of graph theory, and they are very simple than that mentioned in [13]. We think of this is a possible way for solving network transfer delay by using other type of graphical passwords.

"Graphical construction plus number theory" can be realized by graph labellings, and this idea is related with many mathematical conjectures, such as "Every tree is graceful" due to Rosa [11]. The survey article [4] has collected over 1700 papers on research of graph labellings. Motivated from the edge magic graceful labelling [10] and the generalized edge magic total labelling [16], we define a new magic labeling, called generalized edge magic graceful labelling. As known, Kotzig and Rosa [6] defined the edge magic total labelling, and the articles $[3,12,5]$ distributed many results on the edge magic total labelling. MacDougall et al. [7] first defined the vertex magic total labelling, many results on researching the vertex magic total labelling can be found in $[9,8,1]$.

\subsection{An example}

We present an example for realizing the idea of "graphical construction plus number theory" here. A file was encrypted by a lock shown in Fig-2 (f) (or another lock is shown in Fig3 (i)). The lock, Fig-2 (f), must be opened by two keys. Bob was given a graphical construction shown in Fig-1 (a) for making a key shown in Fig-1(c). Bob labelled seven vertices shown in Fig-1 (b), and then he labelled six edges by a rule that each edge is labelled with the absolute value of difference of two labels assigned to two vertices of this edge to finish a complete key shown in (c). Alice was given a graphical construction shown in Fig-1 (a) for making another key shown in Fig-2 (e), she labelled seven vertices shown in Fig-2 (d), and then she labelled six edges, by a rule that each edge is labelled with the absolute value of difference of two labels assigned to two vertices of this edge, to produce a key shown in Fig-2 (e).

Is not difficult to see another group of two keys and another lock are shown in Fig-3.

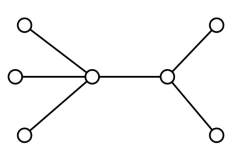

(a)

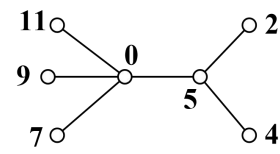

(b)

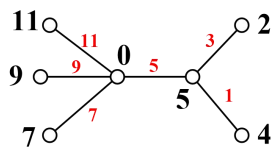

(c)
Figure 1: Bob was given a graphical construction (a) for making a key (c), and he labelled seven vertices shown in (b), and then he labelled six edges shown in (c).

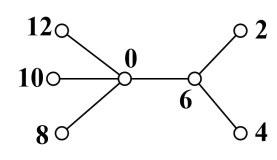

(d)

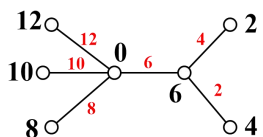

(e)

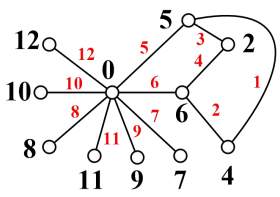

(f)
Figure 2: Alice was given a graphical construction shown in Fig-1 (a) for making another key (e), she labelled seven vertices shown in (d), and then he labelled six edges shown in (e); the lock is shown in (f) 


\subsection{Preliminary}

We use the standard terminology and notation of graph theory here, and the graphs mentioned are finite, undirected, no loops and no multiple edges, other terminology and notation not introduced can be found in [2]. For the purpose of stating quickly, the short hand symbol $[m, n]$ stands for an integer set $\{m, m+1, m+2, \ldots, n\}$, where $m$ and $n$ are non-negative integers with respect to $0 \leq m<n$; and $[s, t]^{o}$ is an odd integer set $\{s, s+2, \ldots, t\}$ with odd numbers $s<t$.

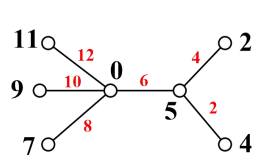

(g)

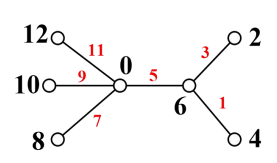

(h)

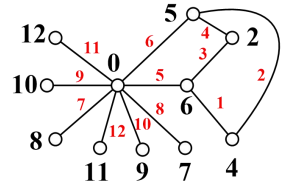

(i)
Figure 3: Another group of two keys shown in $(\mathrm{g})$ and $(\mathrm{h})$; another lock is shown in (i).

A $(p, q)$-graph $G$ has $|V(G)|=p$ vertices and $|E(G)|=q$ edges; $G$ has a proper mapping $f$ from $V(G)$ (or $E(G), V(G) \cup E(G)$ ) to a non-negative integer set $[m, n]$ such that $f(u) \neq f(v)$ for any pair of vertices $u, v$ (or any pair of edges, or any pair of elements), we call $f$ a graph labelling of $G$ hereafter. Write $f(V(G))=\{f(u): u \in V(G)\}$ and $f(E(G))=\{f(u v): u v \in E(G)\}$ in the following discussion.

Definition 1. ([4, 17]) Suppose that a $(p, q)$-graph $G$ admits a mapping $f: V(G) \rightarrow[0, q]$, and the edge label set is defined by $f(E(G))=\{f(u v)=|f(u)-f(v)|: u v \in E(G)\}$. If $f(u) \neq f(v)$ for any pair of vertices $u, v$ of $G$, and $f(E(G))=[1, q]$, then we say $f$ is a graceful labeling of $G$, and $G$ is a graceful graph. Furthermore, if $G$ is a bipartite graph with bipartition $(X, Y)$ such that $\max \{f(x): x \in X\}<\min \{f(y): y \in Y\}$ (or $f(X)<f(Y)$ for short), we call $f$ a set-ordered graceful labeling of $G$.

Definition 2. Suppose that $G$ is a connected $(p, q)$-graph. If there exists a non-negative integer $k$ such that $G$ has a bijection $f: V(G) \cup E(G) \rightarrow[1, p+q],|f(u)+f(v)-f(u v)|=k$ holds true for any edge $u v \in E(G)$, we then call $f$ an edge magic graceful labelling (EMGL) of $G, k$ a magic constant. Furthermore, if $G$ is a bipartite graph with bipartition $(X, Y)$, and $f$ satisfies $\max \{f(x): x \in X\}<\min \{f(y): y \in Y\}$ (or $f(X)<f(Y)$ hereafter) and $f(V(G))=[1, p]$, we call $f$ a super set-ordered edge-magic graceful labelling (super set-ordered EMGL).

The dual labelling $h$ of the labelling $f$ is defined as $h(x)=M+1-f(x)$ for each element $x \in V(G) \cup E(G)$, where $M=$ $\max \{f(x) \mid x \in V(G) \cup E(G)\}$. Clearly, $h$ is an edge magic graceful labelling with magic constant $M+1-k$. We define a new graph labelling in the following definition.

Definition 3. Suppose that $G$ is a connected $(p, q)$-graph. If there exists a non-negative integer $k$ such that $G$ has a bijection $f: V(G) \cup E(G) \rightarrow[1,2 q+1]$, and the form $\mid f(u)+f(v)$ $-f(u v) \mid=k$ holds true for any edge $u v \in E(G)$. Then $f$ is called a generalized edge magic graceful labelling (or GEMGL for short) of $G, k$ a magic constant. If $G$ is a bipartite graph with bipartition $(X, Y)$, and $f$ satisfies $\max \{f(x): x \in X\}<\min \{f(y)$ : $y \in Y\}($ or $f(X)<f(Y)$ hereafter) and $f(V(G))=[1, q+1]$, we call $f$ is a super generalized set-ordered edge-magic graceful labelling (super generalized set-ordered EMGL).

\section{Main theorems for model algorithms}

First of all, we will build up a connection between graceful labellings and generalized edge magic graceful labellings.

Lemma 1. A connected bipartite graph is a set-ordered graceful graph if and only if it admits a super generalized edge magic graceful labelling.

Proof. Suppose that $H$ is a connected bipartite $(p, q)$-graph, its bipartition is $(X, Y)$, where $X=\left\{x_{i}: i \in[1, s]\right\}, Y=\left\{y_{i}: i \in[1, t]\right\}$, $s+t=p$.

The proof of "if". Suppose that $f$ is a set-ordered graceful labelling of $H$ such that $f(X)<f(Y)$, so $f\left(x_{i}\right)=i-1$ with $i \in[1, s]$, and $f\left(y_{j}\right)=s+j$ with $j \in[1, t]$, as well as $f\left(x_{s}\right)<f\left(y_{1}\right)$. Notice that $f\left(x_{i} y_{j}\right)=f\left(y_{j}\right)-f\left(x_{i}\right)=(s+j)-(\mathrm{i}-1) \in[1, q]$ for any edge $x_{i} y_{j} \in E(H)$. We can define a new labelling $g$ of $H$ as follows: Let $g\left(x_{i}\right)=f\left(x_{i}\right)+1, i \in[1, s], g\left(y_{j}\right)=q+1+f\left(y_{1}\right)-f\left(y_{j}\right), j \in[1, t]$; and $g\left(x_{i} y_{j}\right)=M+m+q+1-f\left(x_{i} y_{j}\right)$ for each edge $x_{i} y_{j} \in E(H)$, where $M=\max f(E(H)), m=\min f(E(H))$. Obviously, $\mathrm{g}\left(x_{i}\right) \in\left[1, x_{s}+1\right]$, $g\left(y_{j}\right) \in\left[x_{s}+2, q+1\right]$ and $g\left(x_{i} y_{j}\right) \in[q+2,2 q+1]$. Moreover, we get

$$
\begin{aligned}
& \left|g\left(x_{i}\right)+g\left(y_{j}\right)-g\left(x_{i} y_{j}\right)\right|=\mid f\left(x_{i}\right)+1+q+1+f\left(y_{1}\right)-f\left(y_{j}\right) \\
& -(M+m)-(q+1)+f\left(x_{i} y_{j}\right) \mid \\
= & \left|f\left(x_{i}\right)+1+f\left(y_{1}\right)-f\left(y_{j}\right)-(M+m)+\left[f\left(y_{j}\right)-f\left(x_{i}\right)\right]\right| \\
= & \left|1+f\left(y_{1}\right)-(M+m)\right| \\
= & k .
\end{aligned}
$$

It is easy to see that $k$ is a constant. Thereby, we claim that $\mathrm{g}$ is a super generalized edge magic graceful labelling of $H$.

The proof of "only if". Suppose that the graph $\mathrm{H}$ has a super generalized edge magic graceful labelling $h$, such that $1 \leq h\left(x_{i}\right)<h\left(x_{i+1}\right), i \in[1, s-1] ; h\left(x_{s}\right)<h\left(y_{1}\right), h\left(y_{j}\right)<h\left(y_{j+1}\right) \leq$ $q+1, j \in[1, t-1]$; and

$$
h\left(x_{i} y_{j}\right)=q+1-h\left(y_{1}\right)+h\left(x_{i}\right)+h\left(y_{j}\right) \in[q+2,2 q+1]
$$

for any edge $x_{i} y_{j} \in E(H)$. We compute the vertex label set $h(V(H)) \subset[1, q+1]$, the edge label set $h(E(T))=[q+2,2 q+1]$. We define another labelling $\alpha$ of $H$ in the way: $\alpha\left(x_{i}\right)=h\left(x_{i}\right)-1$, $i \in[1, s]$; and $\alpha\left(y_{j}\right)=q+h\left(y_{1}\right)-h\left(y_{j}\right), j \in[1, t]$; and $\alpha\left(x_{i} y_{j}\right)=2 q^{+}$ $2-h\left(x_{i} y_{j}\right)$. Because

$$
\begin{aligned}
& \alpha\left(y_{j}\right)-\alpha\left(x_{i}\right)=q+h\left(y_{1}\right)-h\left(y_{j}\right)-\left[h\left(x_{i}\right)-1\right] \\
= & q+1+h\left(y_{1}\right)-h\left(y_{j}\right)-h\left(x_{i}\right) \\
= & q+1+h\left(y_{1}\right)+(q+1)-h\left(y_{1}\right)-h\left(x_{i} y_{j}\right) \\
= & 2 q+2-h\left(x_{i} y_{j}\right) \\
= & \alpha\left(x_{i} y_{j}\right),
\end{aligned}
$$

so we have $\left\{\alpha\left(x_{i}\right): i \in[1, s]\right\} \subset\left[0, w_{s}-1\right],\left\{\alpha\left(y_{j}\right): j \in[1, t]\right\} \subset[q$ $\left.+z_{1}-z_{t}, q\right]$, where $z_{t}=q+1$ and $w_{s}<z_{1}$. Therefore, we get $\left\{\alpha\left(y_{j}\right)\right.$ : $\mathrm{j} \in[1, t]\} \subset\left[z_{1}-1, q\right]$, and $\alpha\left(x_{i} y_{j}\right) \in[1, q]$, which show $\alpha(V(T)) \subset$ $[0, q]$ and $\alpha(E(T))=[1, q]$. By the deduction above, we can confirm that $\alpha$ is a set-ordered graceful labellong of $H$.

The proof of this lemma is complete.

Theorem 2. Suppose that $H$ is a bipartite graceful graph. The edge symmetric graph $H=\left\langle K_{2} ; H\right\rangle$ admits some super generalized edge magic graceful labellings. 
Proof. Suppose that $H$ is a bipartite $(p, q)$-graph having a graceful labelling $f,(X, Y)$ is the bipartition of $H$, where $X=\left\{x_{i}\right.$ : $\left.i \in\left[1, m_{1}\right]\right\}, Y=\left\{y_{i}: i \in\left[1, m_{2}\right]\right\}$, and $m_{1}+m_{2}=|V(H)|$. We copy $H$ as $H^{\prime}$. So the copy $H^{\prime}$ is bipartite and graceful $(f$ is a graceful labelling of $\left.H^{\prime}\right)$, and has its bipartition $\left(X^{\prime}, Y^{\prime}\right)$, where $X^{\prime}=\left\{x_{i}^{\prime}: i \in\left[1, m_{1}\right]\right\}$ and $Y^{\prime}=\left\{y_{j}^{\prime}: j \in\left[1, m_{2}\right]\right\}$. Join the vertex u_ $0 \in V(H)$ with its imagine vertex $u_{0}^{\prime} \in V\left(H^{\prime}\right)$ by an edge $u_{0} u_{0}^{\prime}$, write the resulting graph as $G$. It is not difficult to see $V(G)=V(H) \cup V\left(H^{\prime}\right), E(G)=E(H) \cup E\left(H^{\prime}\right) \cup\left\{u_{0} u_{0}^{\prime}\right\}$, and $G=\left\langle K_{2} ;\right.$ $H\rangle$ is an edge symmetric graph with bipartition $\left(X \cup Y^{\prime}, Y \cup X^{\prime}\right)$. We define a labelling $h$ of $G$ based on the graceful labelling $\mathrm{f}$ in the way: Let $h\left(x_{i}\right)=f\left(x_{i}\right), h\left(x_{i}^{\prime}\right)=f\left(x_{i}^{\prime}\right)+q+1$ with $i \in\left[1, m_{1}\right]$; and $h\left(y_{j}\right)=f\left(y_{j}\right)+q+1, h\left(y_{j}^{\prime}\right)=f\left(y_{j}^{\prime}\right)$ for $j \in\left[1, m_{2}\right]$. Thereby, we get that $h(u)<h(v)$ for any vertex $u \in X \cup Y^{\prime}$ and vertex $v \in Y \cup X^{\prime}$. Furthermore, it is not hard to compute

$h\left(X \cup Y^{\prime}\right) \subset[0, q]$ and $h\left(X^{\prime} \cup Y\right) \subset[q+1,2 q+1]$.

The label of every edge $x_{i} y_{j} \in E(H) \subset E(G)$ for $i \neq j$ and $i \in\left[1, m_{1}\right]$ as well as $j \in\left[1, m_{2}\right]$ is equal to

$$
\begin{aligned}
h\left(x_{i} y_{j}\right) & =\left|h\left(y_{j}\right)-h\left(x_{i}\right)\right|=f\left(y_{j}\right)+q+1-f\left(x_{i}\right) \\
& =q+1+\left[f\left(y_{j}\right)-f\left(x_{i}\right)\right]=q+1+f\left(x_{i} y_{j}\right) .
\end{aligned}
$$

On the other hands, each edge $x_{i}^{\prime} y_{j}^{\prime} \in E\left(H^{\prime}\right) \subset E(G)$ for $i \neq j$, $i \in\left[1, m_{1}\right]$, and $j \in\left[1, m_{2}\right]$ holds

$$
\begin{aligned}
h\left(x_{i}^{\prime} y_{j}^{\prime}\right) & =\left|h\left(y_{j}^{\prime}\right)-h\left(x_{i}^{\prime}\right)\right| \\
& =f\left(x_{i}^{\prime}\right)+q+1-f\left(y_{j}^{\prime}\right)=q+1-\left[f\left(y_{j}\right)-f\left(x_{i}\right)\right] \\
& =q+1-f\left(x_{i} y_{j}\right) .
\end{aligned}
$$

For these edges $x_{i} x_{i}^{\prime}$ and $y_{j} y_{j}^{\prime}$ of $G$, we can compute $f\left(x_{i} x_{i}^{\prime}\right)=q+1, f\left(y_{j} y_{j}^{\prime}\right)=q+1$. The above forms show $h(E(G))=[1$, $2 q+1]$. Thereby, we conclude that $h$ is really a set-ordered graceful labelling of $G$. By Lemma 1, the result of this theorem is true.

Theorem 3. Suppose that disjoint bipartite graphs $H_{1}, H_{2}, \ldots$, $H_{m}$ have set-ordered graceful labellings. Then there are vertices $u_{j} \in V\left(H_{j}\right)$ for $j \in[1, m]$ such that the graph $G$ obtained by joining vertex $u_{j} \in V\left(H_{j}\right)$ with the vertex $u_{j+1} \in V\left(H_{j+1}\right)$ with $(j \in[1, m-1])$ by an edge has a super generalized edge magic graceful labelling.

Proof. Suppose that $f_{k}$ is a set-ordered graceful labelling of the $\left(p_{k}, q_{k}\right)$-graph $H_{k}$ with bipartition $\left(H_{k}, H_{k}\right)$ for $k \in[1, m]$, such that $f_{k}\left(X_{k}\right)<f_{k}\left(Y_{k}\right)$, where $X_{k}=\left\{x_{k, i}: i \in\left[1, s_{k}\right]\right\}, Y_{k}=\left\{y_{k, j}\right.$ : $\left.j \in\left[1, t_{k}\right]\right\}$ and $s_{k}+t_{k}=p_{k}$. By the proof of Lemma 1, each $H_{k}$ has a super generalized edge magic graceful labelling $g_{k}$ with $k \in[1, m]$. We set $g_{k}\left(x_{k, i}\right)=f_{k}\left(x_{k, i}\right)+1$ for $i \in\left[1, s_{k}\right]$, and $g_{k}\left(y_{k, j}\right)=q_{k}+1+f_{k}\left(y_{k, 1}\right)-f_{k}\left(y_{k, j}\right)$ with $j \in\left[1, t_{k}\right]$. Each edge $x_{k, i} y_{k, j} \in E\left(H_{k}\right)$ holds

$$
\begin{aligned}
& g_{k}\left(x_{k, i} y_{k, j}\right)=M_{k}+m_{k}+q_{k}+1+f_{k}\left(x_{k, i} y_{k, j}\right) \\
= & M_{k}+m_{k}+q_{k}+1+f_{k}\left(y_{k, j}\right)-f_{k}\left(x_{k, i}\right),
\end{aligned}
$$

where $M_{k}=\max f_{k}\left(E\left(H_{k}\right)\right), m_{k}=\min f_{k}\left(E\left(H_{k}\right)\right)$. Hence, we have

$$
\begin{aligned}
& \left|g_{k}\left(x_{k, i}\right)+g_{k}\left(y_{k, j}\right)-g_{k}\left(x_{k, i} y_{k, j}\right)\right| \\
= & f_{k}\left(x_{k, i}\right)+1+q_{k}+1+f_{k}\left(y_{k, 1}\right) \\
& -f_{k}\left(y_{k, j}\right)-\left[M_{k}+m_{k}+q_{k}+1+f_{k}\left(y_{k, j}\right)-f_{k}\left(x_{k, i}\right)\right]
\end{aligned}
$$

$$
=\left|1+\mathrm{f}\left(y_{k, 1}\right)-\left(M_{k}+m_{k}\right)\right| .
$$

Join the vertex $y_{l, 1} \in V\left(H_{l}\right)$ with the vertex $x_{l+1,1} \in V\left(T_{l+1}\right)$ for $l \in[1, m-1]$ by an edge to produce a new graph, denoted as $G$. Next, we apply the super generalized edge magic graceful labelling $g_{k}$ with $k \in[1, m]$ to define a labelling $g$ of $G$. Let $S=\sum_{r=1}^{m}\left(q_{r}+1\right)$. We, for $k \in[1, m]$, have

(1) For $i \in\left[1, s_{k}\right]$, and $x_{k, i} \in X_{k} \subset V\left(H_{k}\right)$, set

$$
g\left(x_{k, i}\right)=g_{k}\left(x_{k, i}\right)+\sum_{l=1}^{k-1} g_{l}\left(x_{l, s_{l}}\right) ;
$$

(2) For $j \in\left[1, t_{k}\right]$, and $y_{k, j} \in Y_{k} \subset V\left(H_{k}\right)$, let

$$
g\left(y_{k, j}\right)=g_{k}\left(y_{k, j}\right)+\sum_{l=1}^{k-1}\left(q_{l}+1\right)+\sum_{l=k+1}^{m} g_{l}\left(x_{l, s_{l}}\right) ;
$$

(3) Every edge $x_{k, i} y_{k, j} \in E\left(H_{k}\right)$ satisfies

$$
g\left(x_{k, i} y_{k, j}\right)=g_{k}\left(x_{k, i} y_{k, j}\right)+S+\sum_{l=k+1}^{m}\left(q_{l}+1\right)-\left(q_{k}+1\right)
$$

(4) Each edge $x_{l+1,1} y_{l, 1}$ is labelled as

$$
g\left(x_{l+1,1} y_{l, 1}\right)=S+\sum_{l=k+1}^{m}\left(q_{l}+1\right)
$$

with $l \in[1, m-1]$.

We can compute

$$
g\left(x_{k, i}\right) \in\left[1, \sum_{i=1}^{m} g_{i}\left(x_{i, s_{i}}\right)\right], \mathrm{g}\left(y_{k, j}\right) \in\left[\sum_{j=1}^{m} g\left(y_{j, t_{j}}\right), S\right],
$$

$k \in[1, m]$ and $g(E(G))=[S+1,2 S-1]$, as well as $g(V(G)) \subset[1, S]$. Let

$$
\begin{aligned}
K= & \mid 1+f\left(y_{k, 1}\right)-\left(M_{k}+m_{k}\right)+\sum_{l=1}^{m} g_{l}\left(x_{l, s_{l}}\right) \\
& -g_{k}\left(x_{k, s_{k}}\right)-2 \sum_{l=k+1}^{m}\left(q_{l}+1\right) .
\end{aligned}
$$

For each edge $x_{k, i} y_{k, j} \in E\left(H_{k}\right) \subset E(G)$ with $k \in[1, m]$, we have

$$
\begin{aligned}
& \left|\mathrm{g}\left(x_{k, i}\right)-g\left(x_{k, i} y_{k, j}\right)+g\left(y_{k, j}\right)\right| \\
= & \mid g_{k}\left(x_{k, i}\right)+\sum_{l=1}^{k-1} g_{l}\left(x_{l, s_{l}}\right)-g_{k}\left(x_{k, i} y_{k, j}\right) \\
& +S+\sum_{l=k+1}^{m}\left(q_{l}+1\right)-\left(q_{k}+1\right)+g_{k}\left(y_{k, j}\right) \\
& +\sum_{l=1}^{m}\left(q_{l}+1\right)+\sum_{l=k+1}^{m} g_{l}\left(x_{l, s_{l}}\right) \mid \\
= & \mid g_{k}\left(x_{k, i}\right)-g_{k}\left(x_{k, i} y_{k, j}\right)+g_{k}\left(y_{k, j}\right)+\sum_{l=1}^{m} g_{l}\left(x_{l, s_{l}}\right) \\
& \quad-g_{k}\left(x_{k, s_{k}}\right)-2 \sum_{l=k+1}^{m}\left(q_{l}+1\right) \mid \\
= & K .
\end{aligned}
$$

Every edge $x_{k+1,1} y_{k, 1} \in E(G)$ with $k \in[1, m-1]$ holds $\mid g\left(x_{k+1,1}\right)$ $g\left(x_{k+1,1} y_{k, 1}\right)+g\left(y_{k, 1}\right) \mid=K$. Thereby, we have shown that every edge $x y \in E(G)$ holds $|g(x)-g(x y)+g(y)|=K$ true. So we conclude that $g$ is a super generalized edge magic graceful labelling of $G$.

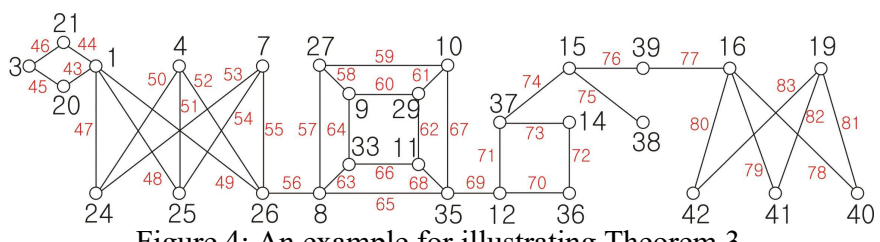

Figure 4: An example for illustrating Theorem 3.

Suppose that $H$ is a graph having super generalized edge magic graceful labellings. Add new vertices $y_{1}, y_{2}, \ldots, y_{t}$ to some vertex $x$ of a graph $H$ by joining each new vertex $y_{i}$ with 
the vertex $x$ by an edge, the resulting graph is denoted by $G$, we say that this process is a firework algorithm.

Theorem 4. Suppose that $H$ is a connected graph having super generalized edge magic graceful labellings. The graph $G$ obtained by doing a firework algorithm do H admits some generalized edge magic graceful labelling.

Proof. Let $h$ be a generalized edge magic graceful labelling of a connected (p, q)-graph $\mathrm{H}$. By the definition, there exists a constant $k \geq 0$ such that each edge $u v \in E(H)$ holds $\mid f(u)+f(v)-$ $f(u v) \mid=k$. We select a vertex $x \in V(H)$ with $t=h(x)-k>0$, and do a firework algorithm to $H$ by adding new vertices $y_{1}, y_{2}, \ldots, y_{t}$ and joining each new vertex $y_{j}$ with the vertex $\mathrm{x}$ for $j \in[1, t]$, so we get a new graph $G$ having vertex set $V(G)=V(H) \cup\left\{y_{1}\right.$, $\left.y_{2}, \ldots, y_{t}\right\}$ and edge set $E(G)=E(H) \cup\left\{x y_{1}, x y_{2}, \ldots, x y_{t}\right\}$. We define a labelling $g$ of $G$ as: Let $g(w)=h(w)$ for $w \in V(H) \cup$ $E(H) ; \mathrm{g}\left(y_{j}\right)=j+(p+q)$ and $\mathrm{g}\left(x y_{j}\right)=j+(p+q)+t$ with $j \in[1, t]$.

Notice that $|h(u)+h(v)-h(u v)|=k$ holds true for each edge $u v \in E(H) \backslash$ subset $E(G)$. For each edge $x y_{j}$, we can compute

$\left|g(x)+g\left(y_{j}\right)-g\left(x y_{j}\right)\right|=|h(x)+[j+(p+q)]-[j+(p+q)+t]|$

$=|h(x)-t|=k$.

Hence, $g$ is really a generalized edge magic graceful labelling of $G$.

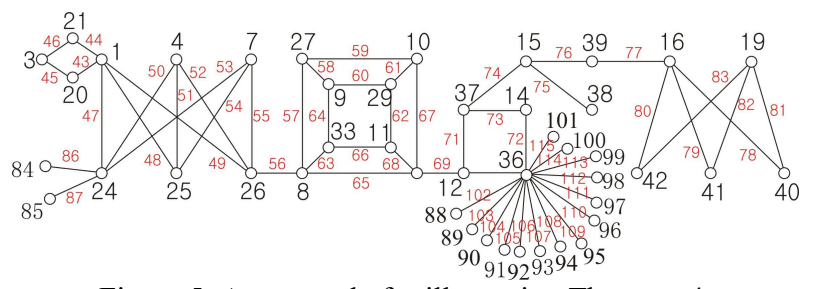

Figure 5: An example for illustrating Theorem 4.

\section{Conclusion}

We have built some network models having generalized edge magic graceful labellings that are new. Our methods to obtain or results can be easily transformed into effective algorithms. The idea of "graphical construction plus number theory" may be a possible way for reducing storage and accelerate computer communication, since graphical passwords introduced in [13] require much more storage space than text based passwords and produce network transfer delay. We can see that "graphical construction plus number theory" is the knowledge based techniques, and people need vast number of simple graphs and easy remembered graph labellings. So more works on this way are waiting for deep and vide research.

\section{Acknowledgements}

The author, Bing Yao, was supported by the National Natural Science Foundation of China under grants No. 61163054, No. 61363060, and No. 616620660.

\section{References}

[1] G. Ali, M. Bača, Y. Lin, and A. Semaničová-Feňovčiková, Super-vertex-antimagic total labelings of disconnected graphs. Discrete Mathematics, 2009, 309 (20):6048-6054.

[2] Bondy J A and Murty U S R. Graph Theory with Applications. New York, 1976.

[3] Ding S, Liu J B, Pei L D. The edge-magic total labeling and the super edge-magic total labeling of $S^{*}$. Journal of Beihua University, 2014.

[4] Joseph A. Gallian. A Dynamic Survey of Graph Labelling. The Electronic Journal of Combinatorics, 14 (2013), \\#DS6.

[5] Hussain M, Baskoro E T, Slamin. On super edge-magic total labeling of banana trees. Utilitas Mathematica, 2009, 79 (7):243-251.

[6] A. Kotzig and A. Rosa. Magic valuations of finite graphs. Canad. Math. Bull. 13 (1970), 451-461.

[7] J.A. MacDougall, M. Miller, Slamin and W.D. Wallis. Vertex-magic total labelings of graphs. Utilitas Math. 61 (2002), 3-21.

[8] Macdougall J A, Miller M, Slamin, et al. Super Vertexmagic Total Labelings of Graphs. Utilitas Math, 2003, 61 (2):222-229.

[9] Mcquillan D. Edge-magic and vertex-magic total labelings of certain cycles. Ars Combinatoria-Waterloo then Winnipeg, 2009, 91:257-266.

[10] Marimuthu G, Kavitha S, Balakrishnan M. Super Edge Magic Graceful Labeling of Generalized Petersen Graphs. Electronic Notes in Discrete Mathematics, 2015, 48:235-241.

[11] Rosa A. On certain valuations of the vertices of a graph. Theory of Graphs, 1967:349-355.

[12] Slamin, Bara M, Lin Y, et al. Edge-magic total labelings of wheels, fans and friendship graphs. Bull.inst.combin.appl, 2002, 35 (35):89-98.

[13] Xiaoyuan Suo, Ying Zhu, G. Scott. Owen, Graphical Passwords: A Survey. In Annual Computer Security Applications Conference (ACSAC), December 2005: 463-472. DOI: 10.1109/CSAC.2005.27 Source: DBLP

[14] Jeff Yan, Ross Anderson, And Alasdair Grant, Password Memorability and Security: Empirical Results. Published By The IEEE Computer Society, 2004, Ieee Security \& Privacy, pp 25-31.

[15] Hongyu Wang, Jin Xu, Bing Yao. Exploring New Cryptographical Construction Of Complex Network Data. 2016 submitted.

[16 ]Wang H, Yao B, Yao M. Generalized edge-magic total labellings of models from researching networks. Information Sciences, 2014, 279:460-467.

[17] Xiangqian Zhou, Bing Yao, Xiang'en Chen and Haixia Tao. A proof to the odd-gracefulness of all lobsters. Ars Combinatoria. 103 (2012), 13-18. 